\title{
Higher Order Fitted Operator Finite Difference Method for Two-Parameter Parabolic Convection-Diffusion Problems
}

\author{
Tesfaye Aga Bullo ${ }^{1 *}$, Gemechis File Duressa ${ }^{2}$, Guy Aymard Degla ${ }^{3}$ \\ ${ }_{1,2}$ Department of Mathematics, Jimma University, Jimma, P.O. Box 378, Ethiopia \\ ${ }^{3}$ Institut De Mathematiques et de sciences physiques, Universit D'Abomey Calavi, Benin \\ E-mail address: tesfayeaga2@gmail.com ${ }^{1 *}$, gammeef@gmail.com ${ }^{1}$, gdegla@gmail.com² \\ ORCID numbers of authors: \\ 0000-0001-6766-4803 ${ }^{1^{*}}, 0000-0003-1889-4690^{2}, 0000-0003-1162-6140^{3}$
}

Received date: 07.11.2019

Accepted date: 24.11 .2019

\begin{abstract}
In this paper, we consider singularly perturbed parabolic convection-diffusion initial boundary value problems with two small positive parameters to construct higher order fitted operator finite difference method. At the beginning, we discretize the solution domain in time direction to approximate the derivative with respect to time and considering average levels for other terms that yields two point boundary value problems which covers two time level. Then, full discretization of the solution domain followed by the derivatives in two point boundary value problem are replaced by central finite difference approximation, introducing and determining the value of fitting parameter ended at system of equations that can be solved by tri-diagonal solver. To improve accuracy of the solution with corresponding higher orders of convergence, we applying Richardson extrapolation method that accelerates second order to fourth order convergent. Stability and consistency of the proposed method have been established very well to assure the convergence of the method. Finally, validate by considering test examples and then produce numerical results to care the theoretical results and to establish its effectiveness. Generally, the formulated method is stable, consistent and gives more accurate numerical solution than some methods existing in the literature for solving singularly perturbed parabolic convection- diffusion initial boundary value problems with two small positive parameters.
\end{abstract}

Keywords: Singularly perturbation parabolic problems, two parameters, fitted operator, accurate solution.

\section{Introduction}

Singularly perturbed parabolic convection-diffusion problems appeared as model in various branches of science and engineering such as modeling of water quality problems in river networks, fluid flow at high Reynold's numbers, convective heat transport problem, drift diffusion equation of semiconductor device modeling, electromagnetic field problem in moving media, financial modeling and turbulence model, one can refer [1, 4, 5, 6, 8, 11]. As stated in book written by Morton [7] and in article presented by Das and Mehrmann [1], singularly perturbed parabolic 
problems describe the transport of solute in groundwater and surface water, the displacement of oil by fluid injection in oil recovery, the movement of aerosols and trace gases in the atmosphere, and describe fluid flow processes in many other applications. Boundary layers occur in the solution of singularly perturbed problems when the singular perturbation parameter multiplies the terms involving the highest order derivatives in the differential equation tends to zero. These boundary layers are neighborhood of the boundary of the domain, where the solution has a very steep gradient.

If one tries to solve singularly perturbed parabolic problems using standard numerical methods applied to solve partial differential equations, then very inaccurate solutions are obtained unless the mesh discretization domain used is extremely small. Even in this context, carful numerical experiments show that the classical computational methods like; standard finite difference or finite element or finite volume methods fail to decrease the maximum point-wise error as the mesh is smaller and smaller; until the mesh size and the perturbation parameter have the same order of magnitude. This contradicts the natural expectation that the error of an acceptable computational method decreases when the mesh is refined. Subsequently, the size of system of algebraic equations will be growing more as the dimension of problem increases. Hence this incorporates the huge computational cost. This drawback motivates researcher to develop and analysis numerical methods which gives accurate numerical solution corresponding to higher order of convergence. Thus, in order to get inexpensive but accurate numerical solution, it will be necessary to develop methods that can handle singular perturbation problems. Hence, numerically solving singularly perturbed parabolic problems depend upon the small positive parameters that affect highest order derivatives of the problem; solution varies rapidly in some parts of the domain and varies slowly in some other parts.

As a result, in the past few decades, various numerical schemes are proposed to solve singularly perturbed parabolic problems with two small positive parameters. For instance, from many few recently developed methods are; parameter-uniform finite element method presented by Kadalbajoo and Yadaw [4], spline difference scheme [12], robust finite difference method [8], robust layer adapted difference method [3] and a parameter-uniform higher order finite difference scheme [2]. All these works concerns singularly perturbed parabolic problems in which perturbation parameters affecting the first and second order derivative terms. While for the perturbation parameter affects only the second order derivative is other type of problem to be discussed separately in other part of our work. These developed methods analyzed very well in different approaches and produce good accurate numerical solution corresponding with first and second order rate of convergence to demonstrate efficiency of the methods.

However, the obtained approximate solution and corresponding order of convergence are not more satisfactory which indicates that yet to solve the stated problem needs develop other numerical methods to produce more accurate numerical solution. Thus, in this work, we formulate, analyze and implement higher order fitted operator finite difference method to solve singularly perturbed parabolic convection-diffusion problems with two small parameters more accurately. 


\section{Formulation of the Method}

We consider the following singularly perturbed parabolic initial boundary value problem (IBVP) on the solution domain $(x, t) \in Q:=\Omega \times(0, T], \Omega=(0,1)$

$$
\varepsilon \frac{\partial 2 u}{\partial x^{2}}+\mu a(x, t) \frac{\partial u}{\partial x}-b(x, t) u(x, t)-\frac{\partial u}{\partial t}=f(x, t)
$$

subject to the initial and boundary conditions

$$
\begin{aligned}
& u(x, 0)=s(x), x \in \bar{\Omega} \\
& u(0, t)=q_{0}(t), u(1, t)=q_{1}(t), t \in[0, T]
\end{aligned}
$$

with two small parameters $0<\varepsilon, \mu<<1$. The coefficient functions $a(x, t), b(x, t)$ and source function $f(x, t)$ are sufficiently regular on $\bar{Q}$ and satisfy $a(x, t) \geq \alpha>0, b(x, t) \geq \beta>0 ; \alpha$ and $\beta$ are real numbers. Also, we assume that sufficient regularity and compatibility conditions imposed on the functions $s(x), q_{0}(t), q_{1}(t)$ and $f(x, t)$, so that a unique solution exists. Problem given by Eqs. (1) and (2) exhibits two boundary layer with different width depending on the relation between the two parameters $\varepsilon$ and $\mu$, one can see refer $[1,2,4]$.

\subsection{Temporal discretization}

To discretize the time variable with uniform step size $k$, so that the time interval $[0, T]$ is partitioned as $0=t_{0}<t_{1}<\ldots<t_{N}=T$ for

$$
t_{n}=n k, \quad k=\frac{T}{N}, n=0,1,2, \ldots, N
$$

Now, at the point $\left(x, t_{n+0.5}\right)$, Eq. (1) can be written as

$$
\varepsilon \frac{\partial 2 u}{\partial x^{2}}\left(x, t_{n+0.5}\right)+\mu a\left(x, t_{n+0.5}\right) \frac{\partial u}{\partial x}\left(x, t_{n+0.5}\right)-b\left(x, t_{n+0.5}\right) u\left(x, t_{n+0.5}\right)=\frac{\partial u}{\partial t}\left(x, t_{n+0.5}\right)+f\left(x, t_{n+0.5}\right)
$$

Using Taylor's series expansion about the point $\left(x, t_{n+0.5}\right)$, we have

$$
\begin{aligned}
& u\left(x, t_{n+1}\right)=u\left(x, t_{n+0.5}\right)+\frac{k}{2} \frac{\partial u}{\partial t} u\left(x, t_{n+0.5}\right)+\frac{k^{2}}{8} \frac{\partial^{2} u}{\partial t^{2}} u\left(x, t_{n+0.5}\right)+\frac{k^{3}}{48} \frac{\partial 3 u}{\partial t 3} u\left(x, t_{n+0.5}\right)+\ldots \\
& u\left(x, t_{n}\right)=u\left(x, t_{n+0.5}\right)-\frac{k}{2} \frac{\partial u}{\partial t} u\left(x, t_{n+0.5}\right)+\frac{k^{2}}{8} \frac{\partial 2 u}{\partial t^{2}} u\left(x, t_{n+0.5}\right)-\frac{k^{3}}{48} \frac{\partial 3 u}{\partial t^{3}} u\left(x, t_{n+0.5}\right)+\ldots
\end{aligned}
$$

which gives

$$
\frac{\partial u}{\partial t}\left(x, t_{n+0.5}\right)=\frac{u\left(x, t_{n+1}\right)-u\left(x, t_{n}\right)}{k}+\tau_{1}=\frac{u_{(x)}^{n+1}-u_{(x)}^{n}}{k}+\tau_{1}
$$


where $\tau_{1}=-\frac{k^{2}}{24} \frac{\partial^{3} u}{\partial t 3} u\left(x, t_{n+0.5}\right) \equiv O\left(k^{2}\right)$

This indicates, the error estimate of time discretization is given by

$$
\left\|E_{n}\right\|_{\infty} \leq C k^{2}
$$

where $\quad C=\frac{1}{24}\left\|\frac{\partial 3 u}{\partial t 3} u\left(x, t_{i+0.5}\right)\right\|, t_{n} \leq t_{i} \leq t_{n+0.5}, \forall i=1,2, \ldots N, \quad$ is a constant independent of parameters $\varepsilon, \mu$ and $k$.

let take the average of the remaining terms in Eq. (4) which is written as

$$
\begin{aligned}
& \varepsilon \frac{\partial^{2} u}{\partial x^{2}}\left(x, t_{n+0.5}\right)+\mu a\left(x, t_{n+0.5}\right) \frac{\partial u}{\partial x}\left(x, t_{n+0.5}\right)-b\left(x, t_{n+0.5}\right) u\left(x, t_{n+0.5}\right)-f\left(x, t_{n+0.5}\right) \\
& \quad=\frac{1}{2}\left(\left\{\varepsilon \frac{d^{2} u^{n+1}}{d x^{2}}+\mu a^{n+1} \frac{d u^{n+1}}{d x}-b_{(x)}^{n+1} u_{(x)}^{n+1}-f_{(x)}^{n+1}\right\}+\left\{\varepsilon \frac{d^{2} u^{n}}{d x^{2}}+\mu a^{n+1} \frac{d u^{n}}{d x}-b_{(x)}^{n} u_{(x)}^{n}-f_{(x)}^{n}\right\}\right)
\end{aligned}
$$

Substituting Eqs. (5) and (7) into Eq. (4) yields linear system of boundary value problems in space at each two time level of the form

$$
\varepsilon \frac{d^{2} u^{n+1}}{d x^{2}}+\mu a^{n+1} \frac{d u^{n+1}}{d x}-\left(b_{(x)}^{n+1}+\frac{2}{k}\right) u_{(x)}^{n+1}+\varepsilon \frac{d^{2} u^{n}}{d x^{2}}+\mu a^{n+1} \frac{d u^{n}}{d x}-\left(b_{(x)}^{n}-\frac{2}{k}\right) u_{(x)}^{n}=f_{(x)}^{n+1}+f_{(x)}^{n}
$$

subject to the boundary conditions at each level

$$
\begin{aligned}
& u\left(0, t_{n+1}\right)=q_{0}\left(t_{n+1}\right), u\left(1, t_{n+1}\right)=q_{1}\left(t_{n+1}\right) \\
& u\left(0, t_{n}\right)=q_{0}\left(t_{n}\right), u\left(1, t_{n}\right)=q_{1}\left(t_{n}\right)
\end{aligned}
$$

The characteristic equation for the homogeneous part with constant coefficients $\alpha$ and $\beta$ of Eq. (8) on $(n+1)^{\text {th }}$ time level is

$$
\varepsilon r^{2}(x)+\mu \alpha r(x)-\left(\beta+\frac{2}{k}\right)=0
$$

Assume it has two real solutions $r_{1}(x)<0$ and $r_{2}(x)>0$ that describe the boundary layers at $x=0$ and $x=1$, respectively. Let

$$
\theta_{1}=-\max _{x \in[0,1]} r_{1}(x) \text { and } \theta_{2}=\min _{x \in[0,1]} r_{2}(x)
$$


The situations of two layers are characterized by the case $\mu<<\varepsilon$ as $\varepsilon \rightarrow 0$, which suggests that $\theta_{1} \approx \theta_{2} \approx \sqrt{\frac{2+k \beta}{\varepsilon k}}$ and we have the layer like to the case $\mu \approx 0$. The other condition, layers arises across in the case where $\varepsilon<<\mu$ as $\mu \rightarrow 0$ yields $\theta_{1} \approx-\max _{x \in[0,1]} \frac{-\mu \alpha}{\varepsilon}=\frac{\mu \alpha}{\varepsilon}$ and $\theta_{2} \approx 0$. Depending on these facts, we have the following two cases:

Case 1: if $\mu<<\varepsilon$ as $\varepsilon \rightarrow 0$, Eq. (1) has two boundary layers of each width $O(\sqrt{\varepsilon})$ then the solution for homogeneous part of Eq.(8) on $(n+1)^{\text {th }}$ time level can be given by

$$
u(x)=A_{1}+B_{1} \exp \left(-\sqrt{\frac{2+k \beta}{\varepsilon k}} x\right)
$$

where $A_{1}, B_{1}$ and $b(x, t) \geq \beta>0$ are real constant numbers.

Case 2: if $\varepsilon<<\mu$ as $\mu \rightarrow 0$, Eq. (1) has two boundary layers of width $O\left(\frac{\varepsilon}{\mu}\right)$ and $O(\mu)$ on the left and right sides respectively, then the solution for homogeneous part of Eq.(8) on $(n+1)^{\text {th }}$ time level can be given by

$$
u(x)=A_{2}+B_{2} \exp \left(-\frac{\mu \alpha}{\varepsilon} x\right)
$$

where $A_{2}, B_{2}$ and $a(x, t) \geq \alpha>0$ are real constant numbers.

Here, most numerical methods gives good accurate solution for case 1 , since $\mu \approx 0$, it has reactiondiffusion parabolic problem property. While for case 2 is challenging to produce good accurate solution. Thus, in this work our focuses to be treat the problem when it is in case 2.

\subsection{The full discrete problem}

Assume that $M$ be positive integer and $\bar{\Omega}^{M}$ denote partition of $[0,1]$ into $M$ subintervals such that $0=x_{0}<x_{1}<\ldots<x_{M}=1$ and $x_{m}=m h, h=\frac{1}{M}, 1=0,1,2, \ldots, M$ then the tensor-product grids on $\bar{Q}^{M, N}$. Undertake the notation $U_{m}^{n} \approx u\left(x_{m}, t_{n}\right)$ and using the central finite difference approximation, Eq. (8) written as

$$
\begin{aligned}
& \varepsilon \frac{U_{m+1}^{n+1}-2 U_{m}^{n+1}+U_{m-1}^{n+1}}{h^{2}}+\mu a_{m}^{n+1} \frac{U_{m+1}^{n+1}-U_{m-1}^{n+1}}{2 h}-\left(b_{m}^{n+1}+\frac{2}{k}\right) u_{m}^{n+1}+ \\
& \varepsilon \frac{U_{m+1}^{n}-2 U_{m}^{n}+U_{m-1}^{n}}{h^{2}}+\mu a_{m}^{n} \frac{U_{m+1}^{n}-U_{m-1}^{n}}{2 h}-\left(b_{m}^{n}-\frac{2}{k}\right) u_{m}^{n}=f_{m}^{n+1}+f_{m}^{n}+\tau_{2}
\end{aligned}
$$


subject to the discrete initial and boundary conditions

$$
\begin{aligned}
& U\left(x_{m}, 0\right)=s\left(x_{m}\right), x_{m} \in \bar{\Omega}^{[0,1]} \\
& U\left(0, t_{n+1}\right)=q_{0}\left(t_{n+1}\right), u\left(1, t_{n+1}\right)=q_{1}\left(t_{n+1}\right), t_{n+1} \in[0, T]^{N}
\end{aligned}
$$

where $\tau_{2}=h 2\left(\mu a_{m}^{n+1}\left(u_{x x x}\right)_{m}^{n+1}+\frac{\varepsilon}{2}\left(u_{x x x x}\right)_{m}^{n+1}\right)-\tau_{1} \equiv O\left(h^{2}+k^{2}\right)$

Let introduce fitting parameter $\sigma$ on the $(n+1)^{t h}$ - level of homogeneous part of Eq. (13), multiply both sides of this equation by $\frac{h}{\mu}$, denote $\rho=\frac{\mu h}{\varepsilon}$ and then evaluate limits both side gives

$$
\frac{\sigma}{\rho}=-\frac{\alpha \lim _{h \rightarrow 0}\left(U_{m+1}^{n+1}-U_{m-1}^{n+1}\right)}{2 \lim _{h \rightarrow 0}\left(U_{m+1}^{n+1}-2 U_{m}^{n+1}+U_{m-1}^{n+1}\right)}
$$

To determine the value of introduced fitting parameter $\sigma$, we consider the discrete form Eq. (12) that leads to

$$
\lim _{h \rightarrow 0} U_{m}^{n+1}=A_{2}+B_{2} \exp (-\alpha m \rho)
$$

Inducing indices from Eq. (16) for $U_{m \pm 1}^{n+1}$ and substituting into Eq. (15), we get

$$
\sigma=\frac{\alpha \rho}{2} \operatorname{coth}\left(\frac{\alpha \rho}{2}\right)
$$

The fitted scheme of Eq. (13) can be written as three term recurrence relation in space direction and two levels in time direction as

$$
E_{m}^{n+1} U_{m-1}^{n+1}-F_{m}^{n+1} U_{m}^{n+1}+G_{m}^{n+1} U_{m+1}^{n+1}=H_{m}^{n+1}, \quad m=1,2, \ldots, M \text { and } n=0,1, \ldots, N
$$

where, $\quad E_{m}^{n+1}=\frac{\varepsilon \sigma}{h^{2}}-\frac{\mu a_{m}^{n+1}}{2 h}, \quad F_{m}^{n+1}=\frac{2 \varepsilon \sigma}{h^{2}}+b_{m}^{n+1}+\frac{2}{k}, \quad G_{m}^{n+1}=\frac{\varepsilon \sigma}{h^{2}}+\frac{\mu a_{m}^{n+1}}{2 h}$

and $H_{m}^{n+1}=f_{m}^{n+1}+f_{m}^{n}-\varepsilon \frac{U_{m+1}^{n}-2 U_{m}^{n}+U_{m-1}^{n}}{h^{2}}-\mu a_{m}^{n} \frac{U_{m+1}^{n}-U_{m-1}^{n}}{2 h}+\left(b_{m}^{n}-\frac{2}{k}\right) u_{m}^{n}$

Hence, scheme developed in Eq. (18) is considered as fitted operator finite difference method to solve the problem in Eqs. (1) and (2). Tri-diagonal system of Eq. (18) with respect to the $x$ direction and the coefficients $E_{m}^{n+1}, F_{m}^{n+1}, G_{m}^{n+1}$ and the right-hand side $H_{m}^{n+1}$ are given that they satisfy the conditions $\left|E_{m}^{n+1}\right|>0,\left|F_{m}^{n+1}\right|>0,\left|G_{m}^{n+1}\right|>0$ and $\left|F_{m}^{n+1}\right|>\left|E_{m}^{n+1}\right|+\left|G_{m}^{n+1}\right|$ at each $(n+1)^{t h}$ level. These situations guarantee that the system is diagonally dominant and it can be solved by tri-diagonal solver. 


\section{Richardson Extrapolation}

This technique is a convergence acceleration technique which involves combination of two computed approximations of a solution. The combination goes out to be an improved approximation. In this work, the truncation terms of the schemes given in Eqs. (5) and (13), we have

$$
\left|u\left(x_{m}, t_{n}\right)-U_{m}^{n}\right| \leq C\left(h^{2}+k^{2}\right)
$$

where $u\left(x_{m}, t_{n}\right)$ and $U_{m}^{n}$ are exact and approximate solutions respectively, $C$ is constant free from mesh sizes $h$ and $k$.

Let $Q_{2 M}^{2 N}$ be the mesh found by dividing each mesh interval in $Q_{M}^{N}$ and symbolize the approximation solution on $Q_{2 M}^{2 N}$ by $\bar{U}_{m}^{n}$. Consider Eq. (19) works for any $h, k \neq 0$, which implies:

$$
u\left(x_{m}, t_{n}\right)-U_{m}^{n} \leq C\left(h^{2}+k^{2}\right)+R_{M}^{N}, \quad\left(x_{m}, t_{n}\right) \in Q_{M}^{N}
$$

So that, it works for any $\frac{h}{2}, \frac{k}{2} \neq 0$ yields:

$$
u\left(x_{m}, t_{n}\right)-\bar{U}_{m}^{n} \leq C\left(\left(\frac{h}{2}\right)^{2}+\left(\frac{k}{2}\right)^{2}\right)+R_{2 M}^{2 N}, \quad\left(x_{m}, t_{n}\right) \in Q_{2 M}^{2 N}
$$

where the remainders, $R_{M}^{N}$ and $R_{2 M}^{2 N}$ are $O\left(h^{4}+k^{4}\right)$.

Combination of inequalities in Eqs. (20) and (21) leads to $3 u\left(x_{m}, t_{n}\right)-\left(4 \bar{U}_{m}^{n}-U_{m}^{n}\right) \approx O\left(h^{4}+k^{4}\right)$ which proposes that

$$
\left(U_{m}^{n}\right)^{e x t}=\frac{1}{3}\left(4 \bar{U}_{m}^{n}-U_{m}^{n}\right)
$$

is also approximation of $u\left(x_{m}, t_{n}\right)$. By means of this approximation to estimate the truncation error, we obtain

$$
\left|u\left(x_{m}, t_{n}\right)-\left(U_{m}^{n}\right)^{e x t}\right| \leq C\left(h^{4}+k^{4}\right)
$$

where $C$ is constant free of mesh sizes $h$ and $k$. Thus, the obtained accelerated method is order four convergent with respect to both independent variables.

\section{Convergence analysis}

The Von Neumann stability technique has been applied to investigate the stability of the developed scheme in Eq. (18), by assuming that the solution of Eq. (18) at the grid point $\left(x_{m}, t_{n}\right)$ is given by: 


$$
U_{m}^{n}=\xi^{n} e^{i m \theta}
$$

where $i=\sqrt{-1}, \theta$ is the real number and $\xi$ is the amplitude factor. Now, substituting Eq. (24) into the homogeneous part of Eq. (18) gives:

$$
\begin{aligned}
E_{m}^{n+1} \xi^{n+1} e^{i(\mathrm{~m}-1) \theta} & -F_{m}^{n+1} \xi^{n+1} e^{i \mathrm{~m} \theta}+G_{m}^{n+1} \xi^{n+1} e^{i(\mathrm{~m}+1) \theta} \\
& =-\varepsilon \frac{\xi^{n} e^{i(\mathrm{~m}+1) \theta}-2 \xi^{n} e^{i \mathrm{~m} \theta}+\xi^{n} e^{i(\mathrm{~m}-1) \theta}}{h^{2}}-\mu a_{m}^{n} \frac{\xi^{n} e^{i(\mathrm{~m}+1) \theta}-\xi^{n} e^{i(\mathrm{~m}-1) \theta}}{2 h}+\left(b_{m}^{n}-\frac{2}{k}\right) \xi^{n} e^{i \mathrm{~m} \theta}
\end{aligned}
$$

This implies that $\xi=\frac{-2 \varepsilon\left(e^{i \theta}-2+e^{-i \theta}\right)-\mu h a_{m}^{n}\left(e^{i \theta}-e^{-i \theta}\right)+2 h 2 b_{m}^{n}}{2 \varepsilon \sigma\left(e^{i \theta}+e^{-i \theta}-2\right)+\mu h a_{m}^{n+1}\left(e^{i \theta}-e^{-i \theta}\right)-2 h 2 b_{m}^{n+1}}$

For sufficiently small mesh size $h$, we have the amplitude factor $\xi=\frac{-1}{\sigma}$ and the condition to be stable is $|\xi| \leq 1$ leads to: $|\xi|=\left|\frac{-1}{\sigma}\right|=\left|-\frac{2}{\alpha \rho} \tanh \left(\frac{\alpha \rho}{2}\right)\right| \leq 1$ which yields $-\frac{\alpha \rho}{2} \leq \tanh \left(\frac{\alpha \rho}{2}\right) \leq \frac{\alpha \rho}{2}$

Since, $\rho=\frac{\mu h}{\varepsilon}$ as $h \rightarrow 0, \tanh \left(\frac{\alpha \rho}{2}\right) \rightarrow 0$. Therefore, $|\xi| \leq 1$.

Hence, the developed scheme in Eq. (18) is stable for any value of mesh sizes. Thus, the developed scheme in Eq. (18) is unconditionally stable by Lax Richtmyer definition [10, 13].

To investigate the consistency of the method, we have considered before extrapolation Eq. (19) and after extrapolation Eq. (23), and then truncation terms vanish as $h \rightarrow 0$ and $k \rightarrow 0$. Hence, the scheme is consistent with the orders of $O\left(h^{2}+k^{2}\right)$ and $O\left(h^{4}+k^{4}\right)$ respectively. Therefore, the constructed scheme is convergent by Lax's equivalence theorem, as stated in books by Smith [12] and Zhilin et. al. [13].

\section{Numerical Results and discussions}

Since the exact solution for such type of problems is not available, the maximum absolute errors at all the mesh points are evaluated for before and after extrapolation using the formula

$$
E_{\varepsilon, \mu}^{M, N}=\max _{0 \leq m \leq M ; 0 \leq n \leq N}\left|u_{m}^{n}-u_{2 m}^{2 n}\right| \text { and } E_{\varepsilon, \mu}^{M, N}=\max _{0 \leq m \leq M ; 0 \leq n \leq N}\left|\left(u_{m}^{n}\right)^{e x t}-\left(u_{2 m}^{2 n}\right)^{\text {ext }}\right| \text { respectively. }
$$

where $u_{m}^{n}$ is approximate solution obtained using a constant space mash size $h$ and time step $k$ and $u_{2 m}^{2 n}$ is also approximate solution produced using space and time step $\frac{h}{2}, \frac{k}{2}$. Also, its solutions obtained by Richardson extrapolation are $\left(u_{m}^{n}\right)^{\text {ext }}$ and $\left(u_{2 m}^{2 n}\right)^{e x t}$. Likewise, we compute the numerical rates of convergence as $R=\frac{\log E_{\varepsilon, \mu}^{M, N}-\log E_{\varepsilon, \mu}^{2 M, 2 N}}{\log 2}$ 
Example 1: Consider the problem

$$
\varepsilon \frac{\partial^{2} u}{\partial x^{2}}+\mu(1+x) \frac{\partial u}{\partial x}-u(x, t)=\frac{\partial u}{\partial t}+16 x^{2}(1-x)^{2},(x, t) \in \Omega:=(0,1) \times(0, T]
$$

subject to the conditions: $u(x, 0)=0, x \in[0,1]$ and $u(0, t)=0=u(1, t), t \in[0, T]$

For this example, the obtained numerical results given below in Tables $1-3$ and Figure 1 .

Example 2: This example corresponds to the following IBVP

$$
\varepsilon \frac{\partial^{2} u}{\partial x^{2}}+\mu\left(1+x(1-x)+t^{2}\right) \frac{\partial u}{\partial x}-(1+5 x t) u(x, t)=\frac{\partial u}{\partial t}+x(1-x)\left(e^{t}-1\right)
$$

subject to the conditions: $u(x, 0)=0, x \in[0,1]$ and $u(0, t)=0=u(1, t), t \in[0,1]$. And numerical results given below in Tables 4 and 5 with Figure 1.

Table 1. Comparison maximum absolute errors for Example 1 at $M=32, k=\frac{0.125}{4}$

\begin{tabular}{ccccc}
\hline$\mu \downarrow \varepsilon \rightarrow$ & $10^{-2}$ & $10^{-4}$ & $10^{-6}$ & $10^{-8}$ \\
\hline Present Method & & & & \\
$10^{-2}$ & $1.3131 \mathrm{e}-06$ & $2.2236 \mathrm{e}-07$ & $2.6487 \mathrm{e}-06$ & $2.6521 \mathrm{e}-06$ \\
$10^{-4}$ & $1.2750 \mathrm{e}-06$ & $2.5782 \mathrm{e}-08$ & $4.9088 \mathrm{e}-10$ & $2.4534 \mathrm{e}-08$ \\
$10^{-8}$ & $1.2746 \mathrm{e}-06$ & $2.4962 \mathrm{e}-08$ & $2.9731 \mathrm{e}-10$ & $2.9926 \mathrm{e}-10$ \\
$10^{-10}$ & $1.2746 \mathrm{e}-06$ & $2.4954 \mathrm{e}-08$ & $2.9747 \mathrm{e}-10$ & $2.9757 \mathrm{e}-10$ \\
Results in [2] & & & & \\
$10^{-2}$ & $1.7212 \mathrm{e}-02$ & $1.7507 \mathrm{e}-02$ & $2.2799 \mathrm{e}-02$ & $2.2801 \mathrm{e}-02$ \\
$10^{-4}$ & $1.7000 \mathrm{e}-02$ & $1.6928 \mathrm{e}-02$ & $1.6913 \mathrm{e}-02$ & $1.6962 \mathrm{e}-02$ \\
$10^{-8}$ & $1.6998 \mathrm{e}-02$ & $1.6923 \mathrm{e}-02$ & $1.6908 \mathrm{e}-02$ & $1.6917 \mathrm{e}-02$ \\
$10^{-10}$ & $1.6998 \mathrm{e}-02$ & $1.6923 \mathrm{e}-02$ & $1.6908 \mathrm{e}-02$ & $1.6917 \mathrm{e}-02$ \\
\hline
\end{tabular}

Table 2. Maximum absolute errors and rate of convergence for Example 1 at $\varepsilon=10^{-12}, T=1$ and

\begin{tabular}{|c|c|c|c|c|c|c|}
\hline$\mu \downarrow N \rightarrow$ & 8 & 16 & 32 & 64 & 128 & 256 \\
\hline \multicolumn{7}{|c|}{ After extrapolation } \\
\hline $10^{-8}$ & $\begin{array}{c}2.0138 \mathrm{e}-07 \\
3.9961\end{array}$ & $\begin{array}{c}1.2620 \mathrm{e}- \\
08 \\
3.9420\end{array}$ & $\begin{array}{c}8.2113 \mathrm{e}- \\
10 \\
3.4733\end{array}$ & $\begin{array}{c}7.3934 \mathrm{e}- \\
11 \\
1.4946\end{array}$ & $\begin{array}{c}2.6237 \mathrm{e}- \\
11 \\
0.9655\end{array}$ & $\begin{array}{c}1.3436 \mathrm{e}- \\
11 \\
---\end{array}$ \\
\hline $10^{-10}$ & $\begin{array}{c}2.0123 \mathrm{e}-07 \\
4.0033\end{array}$ & $\begin{array}{c}1.2548 \mathrm{e}- \\
08 \\
4.0008\end{array}$ & $\begin{array}{c}7.8380 \mathrm{e}- \\
10 \\
4.0003\end{array}$ & $\begin{array}{c}4.8977 \mathrm{e}- \\
11 \\
3.9916\end{array}$ & $\begin{array}{c}3.0789 \mathrm{e}- \\
12 \\
3.9201\end{array}$ & $\begin{array}{c}2.0339 \mathrm{e}- \\
13 \\
---\end{array}$ \\
\hline $10^{-12}$ & $\begin{array}{c}2.0123 \mathrm{e}-07 \\
4.0033\end{array}$ & $\begin{array}{c}1.2548 \mathrm{e}- \\
08\end{array}$ & $\begin{array}{c}7.8377 \mathrm{e}- \\
10\end{array}$ & $\begin{array}{c}4.8983 \mathrm{e}- \\
11\end{array}$ & $\begin{array}{c}3.0536 \mathrm{e}- \\
12\end{array}$ & $\begin{array}{c}2.2882 \mathrm{e}- \\
13\end{array}$ \\
\hline & & & 463 & & & \\
\hline
\end{tabular}
$M=N$ 
Before extrapolation

\begin{tabular}{|c|c|c|c|c|c|c|}
\hline $10^{-8}$ & $\begin{array}{c}3.6002 \mathrm{e}-04 \\
2.0023\end{array}$ & $\begin{array}{c}8.9862 \mathrm{e}- \\
05 \\
2.0006\end{array}$ & $\begin{array}{c}2.2456 \mathrm{e}- \\
05 \\
2.0001\end{array}$ & $\begin{array}{c}5.6135 \mathrm{e}- \\
06 \\
2.0001\end{array}$ & $\begin{array}{c}1.4033 \mathrm{e}- \\
06 \\
2.0000\end{array}$ & $\begin{array}{c}3.5082 \mathrm{e}- \\
07 \\
---\end{array}$ \\
\hline $10^{-10}$ & $\begin{array}{c}3.6002 \mathrm{e}-04 \\
2.0023\end{array}$ & $\begin{array}{c}8.9862 \mathrm{e}- \\
05 \\
2.0005\end{array}$ & $\begin{array}{c}2.2457 \mathrm{e}- \\
05 \\
2.0002\end{array}$ & $\begin{array}{c}5.6136 \mathrm{e}- \\
06 \\
2.0000\end{array}$ & $\begin{array}{c}1.4034 \mathrm{e}- \\
06 \\
2.0000\end{array}$ & $\begin{array}{c}3.5084 \mathrm{e}- \\
07 \\
---\end{array}$ \\
\hline $10^{-12}$ & $\begin{array}{c}3.6002 \mathrm{e}-04 \\
2.0023\end{array}$ & $\begin{array}{c}8.9862 \mathrm{e}- \\
05 \\
2.0005\end{array}$ & $\begin{array}{c}2.2457 \mathrm{e}- \\
05 \\
2.0002\end{array}$ & $\begin{array}{c}5.6136 \mathrm{e}- \\
06 \\
2.0000\end{array}$ & $\begin{array}{c}1.4034 \mathrm{e}- \\
06 \\
2.0000\end{array}$ & $\begin{array}{c}3.5084 \mathrm{e}- \\
07 \\
---\end{array}$ \\
\hline
\end{tabular}

Table 3. Comparison of maximum absolute errors for Example 2 at $\mu=10^{-7}$

\begin{tabular}{ccccc}
\hline$\varepsilon \downarrow$ & $M=64$ & 128 & 256 & 512 \\
& $N=16$ & 32 & 64 & 128 \\
\hline Present Method & & & & \\
$10^{-6}$ & $1.7532 \mathrm{e}-08$ & $1.0945 \mathrm{e}-09$ & $6.0268 \mathrm{e}-10$ & $8.3094 \mathrm{e}-10$ \\
$10^{-7}$ & $1.7532 \mathrm{e}-08$ & $1.0945 \mathrm{e}-09$ & $6.8464 \mathrm{e}-11$ & $2.6425 \mathrm{e}-11$ \\
$10^{-8}$ & $1.7532 \mathrm{e}-08$ & $1.0945 \mathrm{e}-09$ & $6.8466 \mathrm{e}-11$ & $4.2801 \mathrm{e}-12$ \\
$10^{-9}$ & $1.7532 \mathrm{e}-08$ & $1.0945 \mathrm{e}-09$ & $6.8497 \mathrm{e}-11$ & $4.2873 \mathrm{e}-12$ \\
Results in [2] & & & & \\
$10^{-6}$ & $3.8754 \mathrm{e}-5$ & $1.0214 \mathrm{e}-5$ & $2.6170 \mathrm{e}-6$ & $6.6241 \mathrm{e}-7$ \\
$10^{-7}$ & $3.8753 \mathrm{e}-5$ & $1.0214 \mathrm{e}-5$ & $2.6170 \mathrm{e}-6$ & $6.6241 \mathrm{e}-7$ \\
$10^{-8}$ & $3.8753 \mathrm{e}-5$ & $1.0214 \mathrm{e}-5$ & $2.6170 \mathrm{e}-6$ & $6.6241 \mathrm{e}-7$ \\
$10^{-9}$ & $3.8753 \mathrm{e}-5$ & $1.0214 \mathrm{e}-5$ & $2.5461 \mathrm{e}-6$ & $6.6241 \mathrm{e}-7$ \\
Results in [1] & & & & \\
$10^{-6}$ & $9.6949 \mathrm{e}-4$ & $4.9906 \mathrm{e}-4$ & $2.5231 \mathrm{e}-4$ & $1.2824 \mathrm{e}-4$ \\
$10^{-7}$ & $9.8712 \mathrm{e}-4$ & $5.0049 \mathrm{e}-4$ & $2.5485 \mathrm{e}-4$ & $1.2853 \mathrm{e}-4$ \\
$10^{-8}$ & $9.5128 \mathrm{e}-4$ & $5.0026 \mathrm{e}-4$ & $2.5237 \mathrm{e}-4$ & $1.2781 \mathrm{e}-4$ \\
$10^{-9}$ & $9.6746 \mathrm{e}-4$ & $5.0012 \mathrm{e}-4$ & $2.5461 \mathrm{e}-4$ & $1.2803 \mathrm{e}-4$ \\
\hline
\end{tabular}

Table 4. Maximum absolute errors for Example 2 at $\varepsilon=10^{-8}$ and number of intervals $M=N$

\begin{tabular}{|c|c|c|c|c|c|c|}
\hline$\mu \downarrow N \rightarrow$ & 8 & 16 & 32 & 64 & 128 & 256 \\
\hline \multicolumn{7}{|c|}{ After extrapolation } \\
\hline $10^{-8}$ & $2.7269 \mathrm{e}-07$ & $1.7499 \mathrm{e}-08$ & $1.0945 \mathrm{e}-09$ & $6.8442 \mathrm{e}-11$ & $4.2783 \mathrm{e}-12$ & $2.6702 \mathrm{e}-13$ \\
\hline $10^{-10}$ & $2.7269 \mathrm{e}-07$ & $1.7499 \mathrm{e}-08$ & $1.0945 \mathrm{e}-09$ & $6.8442 \mathrm{e}-11$ & $4.2782 \mathrm{e}-12$ & $2.6825 \mathrm{e}-13$ \\
\hline $10^{-12}$ & $2.7269 \mathrm{e}-07$ & $1.7499 \mathrm{e}-08$ & $1.0945 \mathrm{e}-09$ & $6.8441 \mathrm{e}-11$ & $4.2783 \mathrm{e}-12$ & $2.6698 \mathrm{e}-13$ \\
\hline \multicolumn{7}{|c|}{ Before extrapolation } \\
\hline $10^{-8}$ & $1.3949 \mathrm{e}-04$ & $3.5101 \mathrm{e}-05$ & $8.7729 \mathrm{e}-06$ & $2.1959 \mathrm{e}-06$ & $5.4909 \mathrm{e}-07$ & $1.3728 \mathrm{e}-07$ \\
\hline $10^{-10}$ & $1.3949 \mathrm{e}-04$ & $3.5101 \mathrm{e}-05$ & $8.7729 \mathrm{e}-06$ & $2.1959 \mathrm{e}-06$ & $5.4909 \mathrm{e}-07$ & $1.3728 \mathrm{e}-07$ \\
\hline $10^{-12}$ & $1.3949 \mathrm{e}-04$ & $3.5101 \mathrm{e}-05$ & $8.7729 \mathrm{e}-06$ & $2.1959 \mathrm{e}-06$ & $\begin{array}{l}5.4909 \mathrm{e}- \\
07\end{array}$ & $1.3728 \mathrm{e}-07$ \\
\hline
\end{tabular}


Table 5. Order of convergence for Example 2 at $\varepsilon=10^{-8}$ and number of intervals $M=N$

\begin{tabular}{cccccc}
\hline$\mu \downarrow N \rightarrow$ & 8 & 16 & 32 & 64 & 128 \\
\hline After extrapolation & & & & & \\
$10^{-8}$ & 3.9619 & 3.9989 & 3.9992 & 3.9998 & 4.0020 \\
$10^{-10}$ & 3.9619 & 3.9989 & 3.9992 & 3.9998 & 3.9954 \\
$10^{-12}$ & 3.9619 & 3.9989 & 3.9993 & 3.9998 & 4.0022 \\
\multicolumn{1}{l}{ Before extrapolation } & & & & & \\
$10^{-8}$ & 1.9906 & 2.0004 & 1.9982 & 1.9997 & 1.9999 \\
$10^{-10}$ & 1.9906 & 2.0004 & 1.9982 & 1.9997 & 1.9999 \\
$10^{-12}$ & 1.9906 & 2.0004 & 1.9982 & 1.9997 & 1.9999 \\
\hline
\end{tabular}
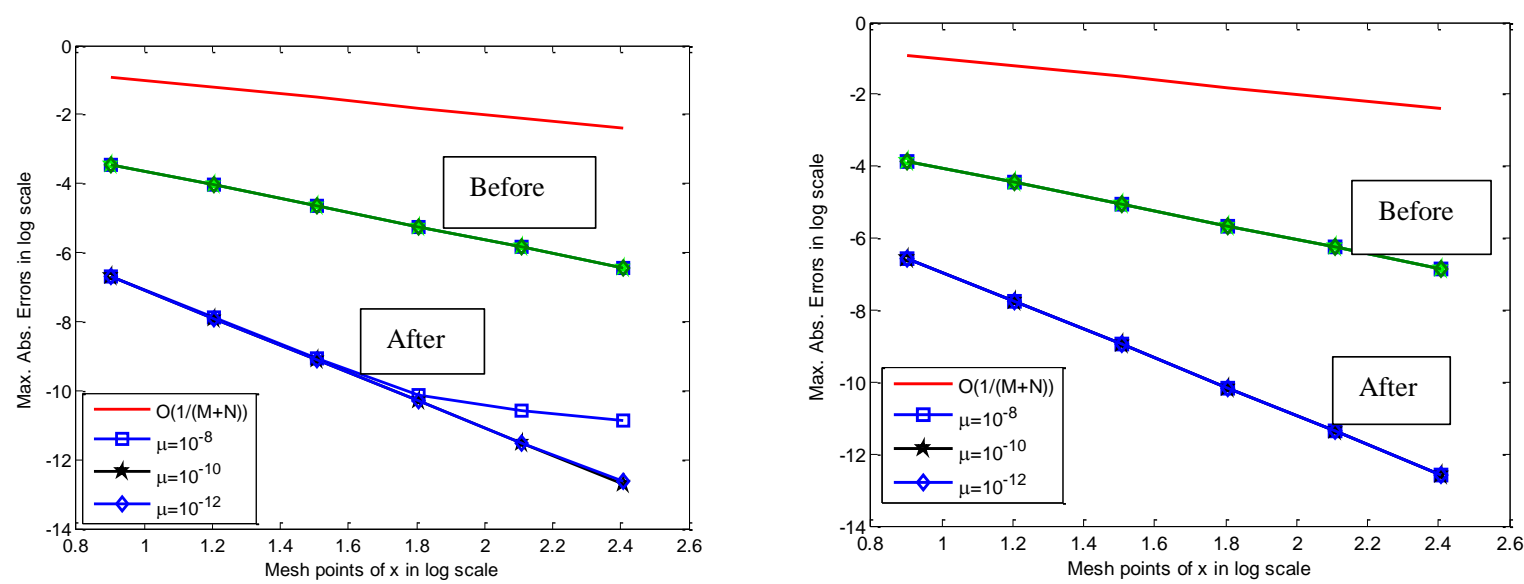

Fig. 1. Log-log plot of maximum absolute errors before and after Richardson extrapolation presented in Table 2 and 4 for Example 1 in the left side and for Example 2 in the right adjacent.

From the results presented in Tables 1 and 3 demonstrates higher order fitted operator finite difference method gives more accurate numerical solution than the existing method. As far results presented in Tables 2, 4 and 5, one can observe that effects of using Richardson extrapolation method to produce more accurate numerical solution corresponding to higher rate of convergence for singularly perturbed parabolic IBVPs with two small positive parameters. Besides, clearly to verify the use of Richardson extrapolation method on numerical scheme to increase accuracy of numerical solution and accelerate order of convergence, one can realize by results confirmed in Figure 1. Moreover, Figure 1 specifies that accuracy of solution increases as number of mesh interval of the domain increases which indicates that convergence of the method.

\section{Conclusion}

The key purpose of this work is to formulate and investigate higher order fitted operator finite difference method to solve singularly perturbed parabolic convection-diffusion IBVPs with two small positive parameters. We first discretize the solution domain in the time direction only which leads to ordinary differential equation with respect to space variable. Secondly, full discretization of domain, derivatives in the differential equations are replaced by central finite difference 
approximation, introduce and determine the value of fitting parameter on $(n+1)^{\text {th }}$ - time level and then the obtained finite difference approximation yields on two-level time direction and three-term recurrence relations in spatial derivatives that can be solved by tri-diagonal solver. Thirdly, applying Richardson extrapolation method to accelerate its rate of convergence from second order to fourth order convergent. Consistency and stability of proposed method have been established very well to guarantee the convergence of the method. Finally, it is validated by considering test examples and displaying numerical results to care the theoretical results and to determine the effectiveness of using the present method. Overall, the developed method is consistent, stable and produces more accurate numerical solution than the existing one for solving singularly perturbed parabolic initial boundary value problems with two small positive parameters.

\section{References}

[1]. Das P. and Mehrmann V., Numerical solution of singularly perturbed convection-diffusionreaction problems with two small parameters, BIT Numer Math, DOI 10.1007/s10543-0150559-8, 2015.

[2]. Gupta V., Kadalbajoo M. K. and Dubey R. K., A parameter uniform higher order finite difference scheme for singularly perturbed time-dependent parabolic problem with two small parameters, International Journal of Computer Mathematics, DOI: 10.1080/00207160.2018.1432856, 2018.

[3]. Jha A. and Kadalbajoo M. K., A robust layer adapted difference method for singularly perturbed two-parameter parabolic problems, International Journal of Computer Mathematics, Taylor and Frances group, 92 1204-1221, 2015.

[4]. Kadalbajoo M. K. and Yadaw A.S., Parameter-uniform finite element method for twoparameter singularly perturbed parabolic reaction-diffusion problems, Int. J. Comput. Methods, 9, 1250047-1 - 1250047-16, 2012.

[5]. Kumar V. and Srinivasan B., A novel adaptive mesh strategy for singularly perturbed parabolic convection diffusion problems, Differ Equ Dyn Syst, DOI 10.1007/s12591-017-0394-2, 2017.

[6]. Miller H. J.J, O’Riordan E. and Shishkin I. G., Fitted numerical methods for singular perturbation problems, Error estimate in the maximum norm for linear problems in one and two dimensions, World Scientific, 1996.

[7]. Morton K. W., Numerical solution of convection-diffusion problems, CRC Press, Taylor and Francis group, 1996.

[8]. Munyakazi J. B., A Robust Finite Difference Method for Two-Parameter Parabolic Convection-Diffusion Problems, An International Journal of Applied Mathematics \& Information Sciences, 9, 2877-2883, 2015.

[9]. Roos G. H., Stynes M.and Tobiska L., Robust numerical methods for singularly perturbed differential equations, Convection-Diffusion-Reaction and Flow Problems, Springer Series in Computational Mathematics, 2008. 
[10]. Smith G. D., Numerical solution of partial differential equations, Finite difference methods, Third edition, Oxford University Pres, 1985.

[11]. Suayip Y. and S. Niyazi S., Numerical solutions of singularly perturbed one-dimensional parabolic convection-diffusion problems by the Bessel collocation method, Applied Mathematics and Computation, 220, 305-315, 2013.

[12]. Zahra W. K., El-Azab M. S. and El Mhlawy A. M. (2014), Spline difference scheme for twoparameter singularly perturbed partial differential equations, J. Appl. Math. and Informatics, $32,185-201,2014$.

[13]. Zhilin L., Qiao Z. and Tang T. Numerical solution of differential equations, Introduction to finite difference and finite element methods, printed in the United Kingdom by Clays, 2018. 